\title{
Effects of hypoxic-ischemic pre-treatment on microvesicles derived from endothelial progenitor cells
}

\author{
WEN ZENG, QIAOLING LEI, JIAO MA and RONG JU \\ Department of Neonatology, Chengdu Women's and Children's Central Hospital, School of Medicine, \\ University of Electronic Science and Technology of China, Chengdu, Sichuan 611731, P.R. China
}

Received December 28, 2018; Accepted December 19, 2019

DOI: $10.3892 /$ etm.2020.8468

\begin{abstract}
Endothelial progenitor cells (EPCs) have protective roles in ischemic injury due to their ability to improve endothelial function and modulate angiogenesis. Microvesicles (MVs) are small membrane particles released by various cell types, including EPCs, which affect various target cells by transferring carried genetic information, including microRNAs (miRNAs/miRs). Depending on the stimuli and cell types, MVs exert different functions. In the present study, oxygen-glucose deprivation (OGD) was used to mimic ischemic-hypoxic (HI) insult, where the effects of $\mathrm{HI}$ insult on EPC-derived MVs (EPC-MVs) were subsequently investigated. OGD induced $\mathrm{Ca}^{2+}$ influx in EPCs and increased the release of EPC-MVs compared with normoxic conditions. In addition, MVs prepared from EPCs cultured under normoxic conditions or OGD conditions (OGD-EMVs) had the ability to stimulate the proliferation of EPCs. Furthermore, OGD-EMVs induced stronger effects on proliferation, which may be associated with the upregulation of miR-210 in EPC-MVs. In conclusion, the present results indicated that $\mathrm{HI}$ insult promoted the release of MVs from EPCs and upregulated miR-210 in MVs, leading to positive modulation of the proliferation of EPCs cultured under normoxic conditions.
\end{abstract}

Correspondence to: Dr Wen Zeng, Department of Neonatology, Chengdu Women's and Children's Central Hospital, School of Medicine, University of Electronic Science and Technology of China, 1617 Riyue Avenue, Chengdu, Sichuan 611731, P.R. China E-mail: zengwen831@163.com

Abbreviations: EPC, endothelial progenitor cell; MV, microvesicle; EPC-MV, EPC-derived microvesicle; miRNA, microRNA; HI, hypoxia-ischemia; OGD, oxygen-glucose deprivation; CCK-8, Cell Counting Kit-8; RT-qPCR, reverse transcription-quantitative PCR

Key words: endothelial progenitor cell, microvesicle, cell viability, hypoxic-ischemic, miR-210

\section{Introduction}

Endothelial progenitor cells (EPCs) are able to mobilize into peripheral blood, home in to an injured area and differentiate into endothelial cells (ECs), thereby contributing to the improvement of endothelial function (1-3). Previous studies have indicated that EPCs are able to exert a protective effect in experimental disease models, including hindlimb ischemia, myocardial infarction and kidney injury (4-8).

Microvesicles (MVs) are small membrane particles 0.1-1 $\mu \mathrm{m}$ in size (9). In response to different stimuli, MVs are released by various cell types, including EPCs, and deliver proteins and genetic information, including mRNA and microRNA (miRNA/miR), to recipient cells, representing a novel method of cell-to-cell communication (10-12). Recent studies have reported that MVs released from different stimuli exert different functions at recipient cells $(13,14)$.

Numerous mammalian organs are vulnerable to hypoxic-ischemic (HI) insult. HI exposure induces a series of changes in mammalian cells that remain incompletely understood. In the short term, HI adaptations are important for improving survival and function, and may be essential for optimal function.

However, to date, the effects of different pathological stimuli on EPC-derived MVs have remained to be fully determined. Oxygen-glucose deprivation (OGD) is a commonly used model to mimic $\mathrm{HI}$ in vitro (15). The present study aimed to investigate the potential effects of $\mathrm{HI}$ on EPC-derived MVs (EPC-MVs) by using OGD culture.

\section{Materials and methods}

Culture of EPCs. EPC culture and characterization was performed as previously described $(16,17)$. In brief, mononuclear cells were isolated from the spleens of Sprague Dawley (SD) rats ( $\mathrm{n}=6$; male; age, 12 weeks; weight, 320-380 g; Third Military Medical University, Chongqing, China). The cells were counted and seeded on fibronectin-coated 24-well plates (BD Biosciences) at $1 \times 10^{6}$ cells/well and then grown in EC basal medium 2 (EBM-2; Gibco; Thermo Fisher Scientific, Inc.), supplemented with $5 \%$ fetal bovine serum (FBS; Invitrogen; Thermo Fisher Scientific, Inc.) containing an EPC growth cytokine cocktail (Gibco; Thermo Fisher Scientific, Inc.) in a $5 \% \mathrm{CO}_{2}$ incubator at $37^{\circ} \mathrm{C}$. After 3 days, 
any non-adherent cells were removed by rinsing three times with PBS. Thereafter, the culture medium was changed every two days.

OGD. In vitro $\mathrm{HI}$ insult was simulated using OGD methods. In brief, the cells were washed three times with glucose-free medium $\left(125 \mathrm{mM} \mathrm{NaCl}, 2.8 \mathrm{mM} \mathrm{KCl}, 1.5 \mathrm{mM} \mathrm{MgCl}_{2}, 0.05 \mathrm{mM}\right.$ $\mathrm{MgSO}_{4}, 2 \mathrm{mM} \mathrm{CaCl}_{2}, 0.83 \mathrm{mM} \mathrm{NaH}_{2} \mathrm{PO}_{4}, 24 \mathrm{mM} \mathrm{NaHCO}_{3}$ and $2 \mathrm{mM}$ HEPES) prior to oxygen removal and placed in an anaerobic chamber perfused with a gas mixture of humidified $95 \% \mathrm{~N}_{2}$ and $5 \% \mathrm{CO}_{2}$ for $3 \mathrm{~h}$ at $37^{\circ} \mathrm{C}$. Control cells were subjected to three washes to control for the mechanical stress associated with medium changes, but otherwise remained in the control culture medium at $37^{\circ} \mathrm{C}$ in a regular $5 \% \mathrm{CO}_{2}$ and $95 \%$ air incubator.

Preparation of $M V s$. MVs were generated from rat EPCs cultured in OGD culture medium (OGD-MVs) or normoxic-condition culture medium (n-MVs). In brief, EPCs were cultured in 100-mm cell culture dishes. When the cells reached $80 \%$ confluence, they were washed with $\mathrm{PBS}$ and then cultured in fresh culture growth medium (EBM-2) or glucose-deprivation medium under oxygen-deprivation conditions in the absence of serum. The cell medium, from 12 wells as an aliquot, was collected and centrifuged at $300 \mathrm{x} \mathrm{g}$ for $5 \mathrm{~min}$ at $4^{\circ} \mathrm{C}$, followed by $2,000 \mathrm{x}$ g for $15 \mathrm{~min} 4^{\circ} \mathrm{C}$, to remove cells and cell debris. The supernatants were ultracentrifuged at $100,000 \times \mathrm{g}$ (Optima L-80 ultracentrifuge; Beckman Coulter) at $4^{\circ} \mathrm{C}$ for $2 \mathrm{~h}$, and then resuspended in PBS after two washes with PBS, each of which was followed by ultracentrifugation at $100,000 \mathrm{x} \mathrm{g}$ for $1 \mathrm{~h}$ at $4^{\circ} \mathrm{C}$. The protein concentration of MVs was quantified via BCA Protein Assay Kit (Beyotime Institute of Biotechnology).

Flow cytometry. Cultured cells were trypsinized and resuspended in PBS containing $1 \%$ bovine serum albumin (Gibco; Thermo Fisher Scientific, Inc.). Cell suspensions were incubated with different antibodies, including CD133-APC (1:100; cat. no. 130-090-422; Miltenyi Biotec), CD34-FITC (1:50; cat. no. sc-7324; Santa Cruz Biotechnology, Inc.), von Willebrand factor (vWF)-FITC (1:200; cat. no. ab8822; Abcam) and fetal liver kinase (Flk)-1-AF647 (1:50; cat. no. sc-6251 AF647; Santa Cruz Biotechnology, Inc.) for $30 \mathrm{~min}$ at room temperature in the dark. MVs were resuspended and incubated for $30 \mathrm{~min}$ at $4^{\circ} \mathrm{C}$ in the dark with Annexin V-FITC (BD Biosciences). Isotype-matched ( $\mathrm{IgG})$ non-specific antibodies served as negative controls. After incubation, labeled cells or MVs were resuspended with PBS and analyzed using flow cytometry (BD Biosciences).

Transmission electron microscopy. For scanning transmission electron microscopy, MVs were fixed in Karnovsky fixative, dehydrated in alcohol, dried on a glass surface and coated with gold by sputter. The specimens were visualized using a Philips Tecnai-10 transmission electron microscope (Philips).

Intracellular $\mathrm{Ca}^{2+}$ detection. The $\mathrm{Ca}^{2+}$-sensitive indicator Fluo-3/AM (BD Biosciences) was used to determine intracellular $\mathrm{Ca}^{2+}$. The dye-loading buffer contained Fluo-3-AM (dissolved in DMSO and pluronic acid) at a final concentra- tion of $4 \mathrm{mM}$ in serum-free cell culture maintenance medium containing $20 \mathrm{mM}$ HEPES and $2.5 \mathrm{mM}$ probenecid. The cells were incubated with the dye-loading buffer for $\sim 30 \mathrm{~min}$ at $37^{\circ} \mathrm{C}$. $\mathrm{Ca}^{2+}$ levels were determined using a FACScan flow cytometer (BD Biosciences) with an excitation wavelength of $488 \mathrm{~nm}$ and an emission wavelength of $525 \mathrm{~nm}$.

Co-culture assay of EPC-MVs and EPCs. EPC-MVs were labeled with DiI (Sigma-Aldrich; Merck KGaA) according to the manufacturer's protocol. In brief, EPC-MVs were labeled with $10 \mu \mathrm{M}$ DiI in PBS for $10 \mathrm{~min}$ at room temperature. An equal volume of FBS was added to stop the staining reaction. EPC-MVs were then ultracentrifuged and resuspended in culture medium for co-culture experiments, wherein the labeled EPC-MVs were added to EPCs, followed by culture for $24 \mathrm{~h}$ in an incubator $\left(37^{\circ} \mathrm{C}, 5 \% \mathrm{CO}_{2}\right)$. Cell nuclei were stained with DAPI (Sigma-Aldrich; Merck KGaA). The interaction between EPC-MVs and EPCs was examined by fluorescence microscopy (Nikon Corp.).

Cell viability assay. Cell viability was assessed with a Cell Counting Kit-8 (CCK-8; Dojindo). EPCs were seeded into 96-well plates (50,000 cells in $100 \mu \mathrm{l} /$ well). Following co-culture with n-MVs, OGD-MVs and pretreated OGD-MVs by using RNase (Thermo Fisher Scientific, Inc.), $10 \mu \mathrm{l}$ CCK-8 solution was added to each well, according to the manufacturer's protocol. After incubation at $37^{\circ} \mathrm{C}$ for $3 \mathrm{~h}$ in a humidified $\mathrm{CO}_{2}$ incubator, the optical density (OD) for each well was measured using a microplate reader (Bio-Rad Laboratories, Inc.) at a wavelength of $450 \mathrm{~nm}$. The OD values were then used to calculate the cell viability by setting the control as $100 \%$.

Reverse transcription-quantitative $(R T-q) P C R$ analysis of $m i R-210$. miR-210 expression was quantified by RT-qPCR, as described previously (18). Total RNA was extracted from MVs harvested from EPC culture medium using a TRIzol isolation system, according to the instructions of the manufacturer (Invitrogen; Thermo Fisher Scientific, Inc.). Complementary DNA was synthesized using an miScript RT kit (Qiagen). RT-qPCR was performed with specific primers for miR-210 or U6 and a miScript SYBR Green PCR Kit (Qiagen) on a real-time PCR system (Bio-Rad Laboratories, Inc.). U6 was used as the internal control. The primers used are listed in Table I. The thermocycling conditions were: Initial denaturation at $94^{\circ} \mathrm{C}$ for $4 \mathrm{~min}$, followed by a total of 35 cycles of $94^{\circ} \mathrm{C}$ for $20 \mathrm{sec}, 60^{\circ} \mathrm{C}$ for $30 \mathrm{sec}$ and $72^{\circ} \mathrm{C}$ for $30 \mathrm{sec}$. All experiments were run in triplicate and the relative expression of miR-210 was calculated using the $2^{-\Delta \Delta \mathrm{Cq}}$ method (19).

miR-210 overexpression in EPCs. EPCs were transfected with miR210 mimics (sense, 5'-CUGUGCGUGUGACAG CGGCUGA-3' and antisense: 5'-UUGACACGCACACUG UCGCCGA; 20 nM; Western Biomedical Technology) using Lipofectamine $^{\circledR} 2000$ reagent (Invitrogen; Thermo Fisher Scientific, Inc.) for $48 \mathrm{~h}$. In the control group, EPCs were transfected with scrambled miRNA (miRNA-NC; 20 nM; Western Biomedical Technology). Overexpression was then verified by RT-qPCR. The viability of the EPCs was detected using CCK- 8 assay. The control group were EPCs which were trans- 
Table I. Primers used for quantitative PCR analysis.

Gene/primer

direction

Sequence

$\begin{array}{ll}\begin{array}{l}\text { miR-210 } \\ \text { Forward }\end{array} & \text { 5'-GTGCAGGGTCCGAGGT-3' } \\ \text { Reverse } & \text { 5'-CTGTGCGTGTGACAGCGGCTGA-3 } \\ \text { U6 } & \\ \text { Forward } & \text { 5'-CTCGCTTCGGCAGCACA-3' } \\ \text { Reverse } & \text { 5'-AACGCTTCACGAATTTGCGT-3' }\end{array}$

miR, microRNA

fected with $20 \mathrm{nM}$ scrambled miRNA (miRNA-NC; sense, 5'-UUCUCCGAACGUGUGUCACGUTT-3' and antisense, 5'-ACGUGACACGUUCGGAGAATT-3'; Western Biomedical Technology).

Statistical analysis. Values are expressed as the mean \pm standard deviation and analyzed using SPSS v.18.0 software (SPSS, Inc.). Statistical analyses were performed using one-way ANOVA with Tukey's post hoc test for comparison among multiple groups or the Student's t-test for comparison between two groups. $\mathrm{P}<0.05$ was considered to indicate statistical significance. All statistical analyses were performed in a blinded manner.

\section{Results}

Isolation and identification of EPCs. EPCs were isolated from 12 -week-old SD rats and successfully propagated in vitro. Isolated spleen-derived EPCs exhibited a spindle-shaped morphology after 4-7 days of culture (Fig. 1A and B). Although there is currently no specific marker, it is generally agreed that rat EPCs are positive for CD133, CD34, vWF and Flk-1 $(20,21)$. EPCs at passage 5 were identified by flow cytometry using these cell makers. In the present study, 55-95\% of the cells were tested positive for CD133-, CD34-, vWF- or Flk-1 (Fig. 1C-F; Table II). The data confirm that the cells used in the present study were indeed EPCs.

OGD promotes the release of $M V s$. Protein quantification indicated that OGD treatment increased the total protein in MVs harvested from EPC culture medium. A clear increase in the total protein content of MVs was observed after OGD $(485.42 \pm 11.01$ vs. $567.79 \pm 14.89 \mu \mathrm{g} / \mathrm{ml}$ with $\mathrm{n}-\mathrm{MVs}$ and OGD-MVs, respectively; $\mathrm{P}<0.05$; Fig. $2 \mathrm{~A}$ ). In addition, in the images captured via transmission electron microscopy, more MVs were observed in the OGD group compared with those in the normoxic group (Fig. 2B). These results implied that increased numbers of MVs were released by EPCs after OGD treatment.

Previous studies have suggested that $\mathrm{Ca}^{2+}$ influx has a vital role in the process of $\mathrm{MV}$ release and that HI may induce cellular $\mathrm{Ca}^{2+}$ influx (22). To determine whether the release of MVs after OGD pre-treatment is associated with $\mathrm{Ca}^{2+}$ influx, intracellular $\mathrm{Ca}^{2+}$ levels were detected using the


CD34 FITC


Figure 1. Culture and identification of EPCs. (A and B) Morphology of EPCs after (A) 4 days and (B) 7 days of culture, assessed under a light microscope (scale bar, $10 \mu \mathrm{m}$ ); (C-F) EPCs were identified by flow cytometry following labeling with antibodies against (C) CD133, (D) CD34, (E) vWF and (F) Flk-1; isotype $\mathrm{IgG}$ served as a negative control (negative controls are presented in the left-hand panels and EPCs in the right-hand panels). EPC, endothelial progenitor cell; vWF, von Willebrand factor; Flk-1, fetal liver kinase 1.

fluorescent $\mathrm{Ca}^{2+}$ indicator Fluo-3/AM. In the present study, it was indicated that treatment of EPCs with OGD resulted in an apparent $\mathrm{Ca}^{2+}$ fluctuation. As presented in Fig. 3, a clear increase in the fluorescence intensity was observed in the OGD group as compared with that in the normoxic group $(562.75 \pm 27.40$ vs. $1,426.50 \pm 18.34 \%$ in the normoxic and OGD groups, respectively; $\mathrm{P}<0.05)$. The results suggested that the release of MVs from EPCs following OGD treatment was also associated with $\mathrm{Ca}^{2+}$ influx.

OGD treatment induces increased expression of miR210 in MVs. Considering miR-210 is sensitive to hypoxia and is involved in certain pathogeneses associated with hypoxia, miR-210 levels in MVs were examined. As presented in Fig. 4, RT-qPCR analysis revealed that the levels of miR-210 were 
Table II. Ratio of positively labeled cells identified by flow cytometry.

\begin{tabular}{lcc}
\hline Marker & Positive & Negative \\
\hline CD133 & $58.98 \pm 1.98$ & $43.72 \pm 2.05$ \\
CD34 & $71.80 \pm 1.66$ & $30.79 \pm 1.69$ \\
vWF & $82.40 \pm 1.07$ & $19.22 \pm 1.16$ \\
Flk-1 & $95.61 \pm 0.65$ & $2.1 \pm 1.21$ \\
\hline
\end{tabular}

Values are expressed as the mean \pm standard deviation $(n=3)$. vWF, von Willebrand factor; Flk-1, fetal liver kinase 1.



n-MVs
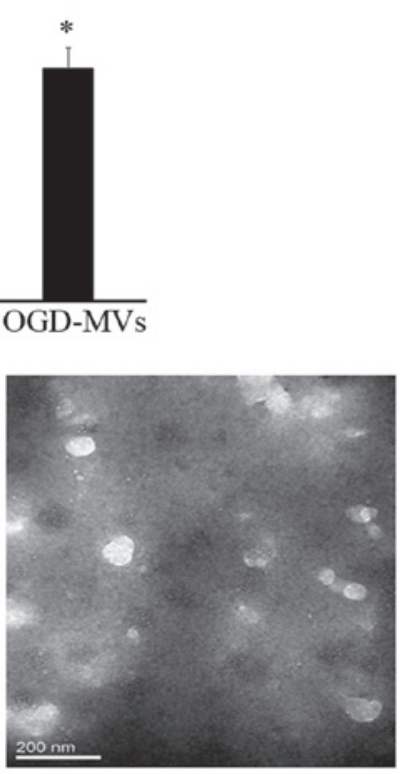

OGD-MVs
Figure 2. OGD treatment increases the release of MVs from EPCs. (A) Tota protein content of $\mathrm{MVs}$ was quantified; ${ }^{*} \mathrm{P}<0.05$ vs. normoxic group. (B) Images of MVs collected from the aliquot of EPCs culture medium using ultracentrifugation in the normoxic group and OGD group, captured by a transmission electron microscope (scale bar, $200 \mathrm{~nm}$ ). MV, microvesicle; OGD, oxygen-glucose deprivation; n-MVs, MVs in normoxic culture; EPC, endothelial progenitor cell.

markedly increased in OGD-MVs as compared with those in the normoxic group. In addition, a time-dependent increase was also observed in miR-210 expression in the OGD group. These results demonstrated that miR-210 may be produced and secreted by EPCs, and packaged further into MVs in response to OGD (HI insult).

$M V s$ incorporated into EPCs after in vitro co-incubation. To observe whether MVs could be incorporated into EPCs, DiI-labeled MVs were added into EPCs culture. After co-incubation of DiI-labeled MVs with EPCs for $24 \mathrm{~h}$, DiI fluorescence was detected in EPCs. Immunofluorescence analysis suggested that the MVs were incorporated into the EPCs (Fig. 5).

$M V$ s promote the proliferation of EPCs. As OGD led to upregulated miR-210 expression in MVs and miR-210 is
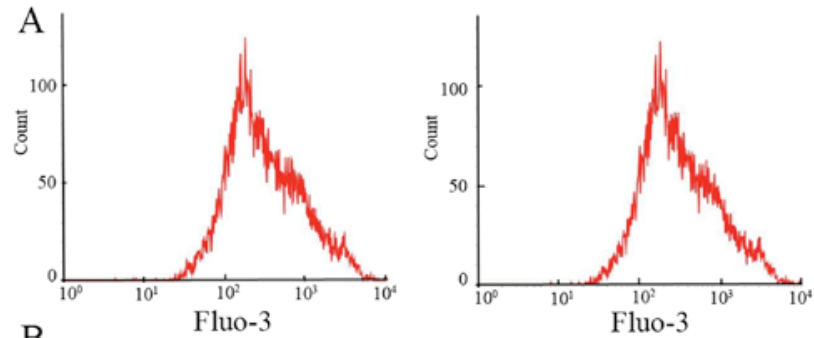

B
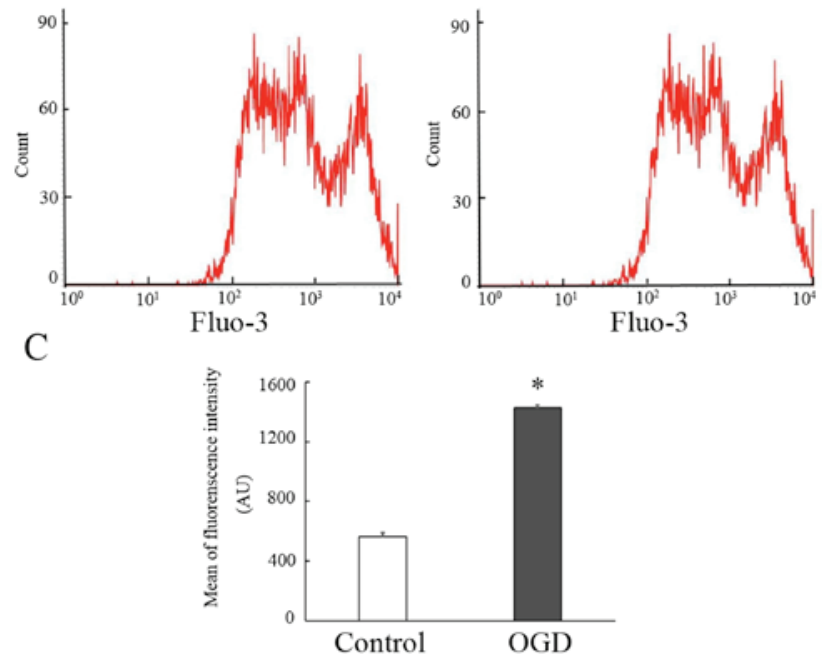

Figure 3. Intracellular $\mathrm{Ca}^{2+}$ levels in endothelial progenitor cells were detected by Fluo-3/AM flow cytometry. (A and B) Flow cytometric analysis of intracellular $\mathrm{Ca}^{2+}$ levels in (A) the normoxic group and (B) OGD group. (C) Histograms of $\mathrm{Ca}^{2+}$ fluorescence intensity in the normoxic group and OGD group. " $\mathrm{P}<0.05$ vs. normoxic group (mean \pm standard deviation, $\mathrm{n}=4$ ). OGD, oxygen-glucose deprivation.



Figure 4. Relative expression of miR-210 as detected by reverse transcription-quantitative PCR. The expression of miR-210 in collected MVs was increased at 2, 4, 12 and $24 \mathrm{~h}$ after treatment with OGD, while there was no significant change in miR-210 expression in the normoxic group. ${ }^{*} \mathrm{P}<0.05$ vs. normoxic group (mean \pm standard deviation, $n=3$ ). MV, microvesicle; miR, microRNA; n-MVs, MVs in normoxic culture; miR, microRNA; OGD, oxygen-glucose deprivation.

associated with cell proliferation, it was presumed that MVs treated with OGD may be involved in modulating the proliferation of EPCs. For this purpose, EPCs were cultured with MVs collected from OGD and normoxic culture media, and it was revealed that co-culture with MVs increased EPC proliferation; the increase in the OGD-MV group was the most significant (Fig. 6). 
A
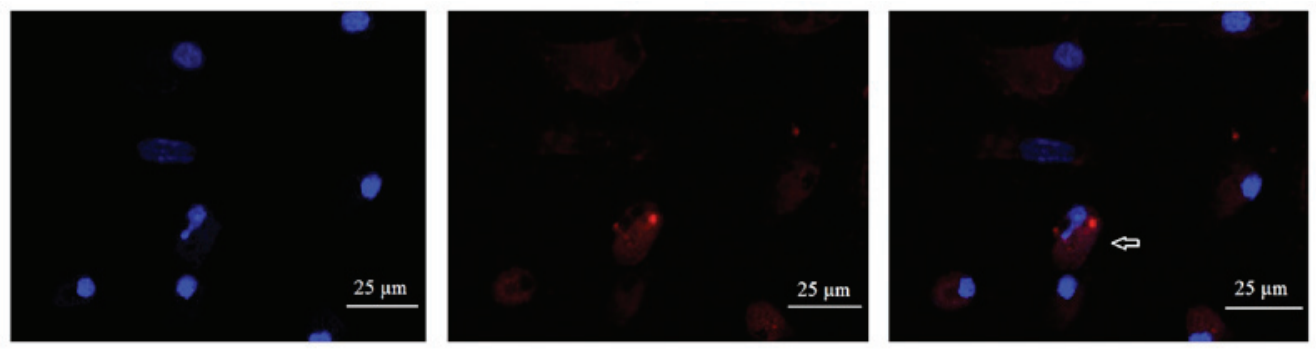

$\mathrm{B}$

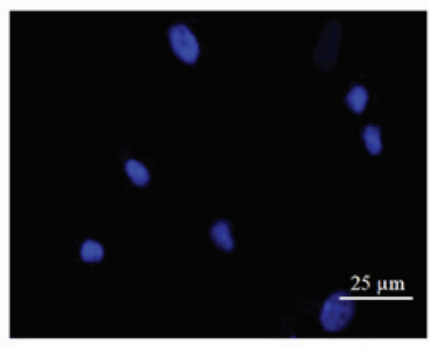

DAPI

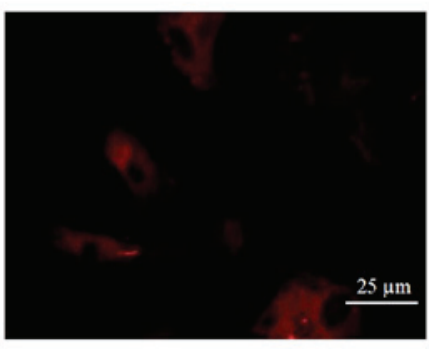

Dil

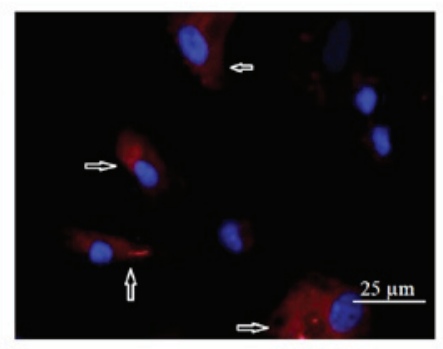

Merge

Figure 5. Microvesicle incorporation into EPCs was observed by fluorescence microscopy. (A) n-MV were incorporated into EPCs; (B) MVs treated with OGD were incorporated into EPCs (blue, DAPI; red, DiI; magnification, x800; scale bar, $25 \mu \mathrm{m}$ ). EPC, endothelial progenitor cell; MV, microvesicle; n-MVs, MVs in normoxic culture; OGD, oxygen-glucose deprivation.



Figure 6. Effects of MVs on EPC proliferation as measured by a Cell Counting Kit- 8 assay. EPCs were co-cultured with MVs released from cells with different pre-treatments. EPCs in the three co-cultured groups exhibited increased proliferation compared with that of monocultured EPCs, and MVs released from the group pre-treated with OGD exhibited the highest proliferative effects, which was counteracted by $\mathrm{RNase}$. ${ }^{*} \mathrm{P}<0.05$ vs. control group, ${ }^{\wedge} \mathrm{P}<0.05$ vs. $\mathrm{n}-\mathrm{MV}$ group, ${ }^{\#} \mathrm{P}<0.05$ vs. OGD-MVs group (mean \pm standard deviation, $\mathrm{n}=4$ ). EPC, endothelial progenitor cell; MV, microvesicle; OGD, oxygen-glucose deprivation; n-MVs, MVs in normoxic culture.
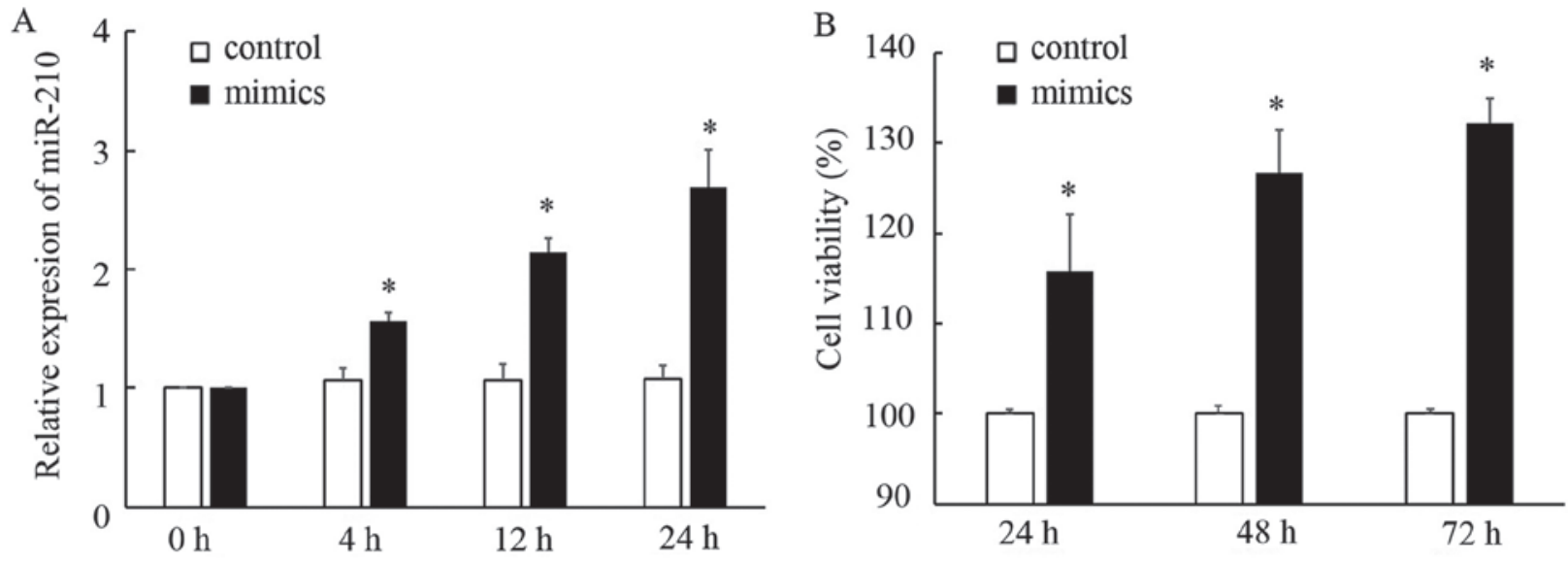

Figure 7. Effects of miR-210 mimics on EPCs. EPCs were transfected with miR-210 mimics. (A) The expression of miR-210 in EPCs after transfection was detected using reverse transcription-quantitative PCR. (B) Proliferation of EPCs after transfection was detected using a Cell Counting Kit- 8 assay. ${ }^{*} \mathrm{P}<0.05$ vs. control group (mean \pm standard deviation, $n=4$ ). EPC, endothelial progenitor cell; miR, microRNA. 
Previous studies have indicated that treatment of MVs with high concentrations of RNase inactivated the RNAs carried by MVs $(10,23)$. In the present study, the involvement of MVs in EPC proliferation was further confirmed by using RNase to pre-treat MVs. With the digestion of RNase, the proliferation of EPCs was visibly decreased as compared with that in the other two co-culture groups lacking pre-treatment with RNase, but it remained slightly higher than that in the control group (Fig. 6).

miR-210 mimics promote the proliferation of EPCs. To further confirm whether miR-210 is involved in EPC proliferation, EPCs were transfected with miR-210 mimics. As presented in Fig. 7, transfection of miR-210 mimics led to overexpression of miR-210 and a significant increase in the proliferation of EPCs, indicating an association between miR-210 and EPC proliferation.

\section{Discussion}

In the present study, MVs derived from EPCs that had been subjected to OGD were obtained. There were three major results: First, it was demonstrated that OGD induced $\mathrm{Ca}^{2+}$ influx in EPCs. Furthermore, it was revealed that OGD increased the release of EPC-MVs compared with that under normoxic conditions. Finally, it was demonstrated that OGD increased the level of miR-210, and transfection of miR-210 mimics into EPC-MVs led to stimulation of EPC proliferation under normal culture conditions.

Therapeutic angiogenesis and vasculogenesis have emerged as promising therapies to treat ischemic diseases $(24,25)$. Ischemic injury is multifactorial in its etiology. One of the factors involves the disruption of vascular integrity, causing vessel vulnerability. It is well known that the endothelium serves as a barrier between vessel walls and the blood, and has an essential role in regulating vascular homeostasis (26). It has been demonstrated that EPCs are able to mobilize in the peripheral blood, home in to an injured area, differentiate into ECs and promote vascular repair. Furthermore, evidence indicates that transplantation of EPCs promotes new vessel formation and alleviates injury to ischemic tissues, suggesting that EPCs actively contribute to angiogenesis and vasculogenesis (27-29).

Transplantation of EPCs is a potential therapy for ischemic diseases (5-8). However, similar to other types of stem cells, certain limitations still exist, including the mode of action, immunogenicity, tumorigenicity, proliferation capacity and overall feasibility of use (30). Further studies on the optimization of the safety and efficacy of stem cell transplantation are required, while recent studies have provided novel insight, including information on MVs.

MVs shed from the surface of viable cells and act as paracrine mediators due to their ability to incorporate into target cells in order to exert their functions. In particular, the therapeutic potential of MVs has been taken into consideration when designing studies. By using a model of kidney injury induced by ischemia-reperfusion, it has been demonstrated that the injection of MSC-derived MVs has a significant protective effect on renal function $(31,32)$. Another study suggested that MVs derived from ECs modulated astrocyte function, blood-brain barrier integrity and cerebral blood flow, indicating MVs may serve as a novel therapeutic target for ischemic stroke (33). The biological functions of MVs are tightly associated with their contents. Contents of MVs are not randomly packaged, but influenced by various factors, including external stimuli. Hence, the package of MVs is a precisely regulated process. RNA inactivation in MVs, achieved by treatment with high concentrations of RNase, is able to reduce the protective effects of MVs $(32,34)$, indicating that genetic information transferred by MVs has a pivotal role in their biological functions.

Endogenous miRNAs have been identified to have essential roles in gene regulation to modulate physiological and pathological processes. miRNAs are short, non-coding RNA molecules of 19-23 nucleotides in length, which function as inhibitors of target gene expression by inducing mRNA degradation or translational repression $(35,36)$. Serval studies have demonstrated that expression of a certain set of miRNAs is upregulated under hypoxia, including miR-210 (37-40). miR-210 is evolutionarily conserved and widely expressed in various cell and tissue types. In specific cell types, including primary vascular ECs, hypoxia induces an augmented expression of miR-210 $(38,41)$. Data on the genetic structure indicate that the promoter region of miR-210 carries a functioning hypoxia response element (HRE), which may be recognized by hypoxia-inducible factor-1 $\alpha$ for the induction of miRNA transcription upon exposure to hypoxia $(42,43)$. Thus, miR-210 is sensitive to hypoxic stimuli and has an important role in modulating hypoxia-induced pathogeneses. A diverse range of genes have been demonstrated to be direct targets of miR-210, including iron-sulfur cluster assembly protein ISCU1/2, fibroblast growth factor receptor-like 1, FLASH/caspase-8 associated protein-2, HOXA3, RAD52 homolog, DNA repair protein and tyrosine kinase ligand ephrin-A3 (41,44-48). Through the suppression of direct target transcripts, miR-210 is involved in modulating the processes of cellular survival, metabolism, proliferation, DNA repair and endothelial angiogenesis (38).

Thus, together with the in vitro results of the present study, it is indicated that HI pre-treatment may activate stronger protective effects of EPC-MVs through increasing the release and elevating the expression of miR-210 in the EPC-MVs. It is possible that the beneficial effects of EPC-MVs induced by HI insult may contribute to injury repair. However, further studies focusing on the detailed effects and mechanisms of the release and contents of EPC-MVs induced by HI insult are required.

In conclusion, the present study demonstrated that treatment with EPC-MVs produced under HI conditions was able to promote the proliferation of EPCs, which was associated with elevated expression of miR-210. The effects of HI insult on EPC-MVs may offer novel therapeutic strategies for tissue injury.

\section{Acknowledgements}

Not applicable.

\section{Funding}

This work was supported by the National Natural Science Foundation of China (grant no. 81401247), Sichuan Science 
and Technology Program (grant nos. 2016JY0126 and 2019YJ0648) and Science and Technology Project of Chengdu, Sichuan Province, China (grant no. 2014-HM01-00348-SF).

\section{Availability of data and materials}

All data generated or analyzed during the present study are included in this published article.

\section{Authors' contributions}

WZ designed the study, performed the experiments and the data analysis and wrote the manuscript. QL performed cell culture, preparation of MVs and transmission electron microscopy. JM performed the flow cytometry and RT-qPCR experiments. RJ collected and analyzed the data and revised the manuscript.

\section{Ethics approval and consent to participate}

All procedures conformed to the local principles of laboratory animal care and were approved by the Institutional Animal Care and Use Committee of Chengdu Women's \& Children's Central Hospital (Sichuan, China; approval no. 2019-31). Precautions were taken to minimize suffering and the number of animals used in each experiment.

\section{Patient consent for publication}

Not applicable.

\section{Competing interests}

The authors declare that they have no competing interests.

\section{References}

1. Szmitko PE, Fedak PW, Weisel RD, Stewart DJ, Kutryk MJ and Verma S: Endothelial progenitor cells: New hope for a broken heart. Circulation 107: 3093-3100, 2003.

2. Sekiguchi H, Ii M, Jujo K, Yokoyama A, Hagiwara N and Asahara T: Improved culture-based isolation of differentiating endothelial progenitor cells from mouse bone marrow mononuclear cells. PLoS One 6: e28639, 2011.

3. Hristov $M$ and Weber C: Endothelial progenitor cells: Characterization, pathophysiology, and possible clinical relevance. J Cell Mol Med 8: 498-508, 2004.

4. Li SC, Acevedo J, Wang L, Jiang H, Luo J, Pestell RG, Loudon WG and Chang AC: Mechanisms for progenitor cell-mediated repair for ischemic heart injury. Curr Stem Cell Res Ther 7: 2-14, 2012.

5. Jujo K, Ii M and Losordo DW: Endothelial progenitor cells in neovascularization of infarcted myocardium. J Mol Cell Cardiol 45: 530-544, 2008.

6. Kwon O, Miller S, Li N, Khan A, Kadry Z and Uemura T: Bone marrow-derived endothelial progenitor cells and endothelial cells may contribute to endothelial repair in the kidney immediately after ischemia-reperfusion. J Histochem Cytochem 58: 687-694, 2010.

7. Yu P,Li Q, Liu Y,Zhang J, Seldeen K and Pang M: Pro-angiogenic efficacy of transplanting endothelial progenitor cells for treating hindlimb ischemia in hyperglycemic rabbits. J Diabetes Complications 29: 13-19, 2015.

8. Teraa M, Sprengers RW, Schutgens RE, Slaper-Cortenbach IC, van der Graaf Y, Algra A, van der Tweel I, Doevendans PA, Mali WP, Moll FL and Verhaar MC: Effect of repetitive intra-arterial infusion of bone marrow mononuclear cells in patients with no-option limb ischemia: The randomized, double-blind, placebo-controlled rejuvenating endothelial progenitor cells via transcutaneous intra-arterial supplementation (JUVENTAS) trial. Circulation 131: 851-860, 2015.
9. Rautou PE and Mackman N: Microvesicles as risk markers for venous thrombosis. Expert Rev Hematol 6: 91-101, 2013.

10. Deregibus MC, Cantaluppi V, Calogero R, Lo Iacono M, Tetta C, Biancone L, Bruno S, Bussolati B and Camussi G: Endothelial progenitor cell derived microvesicles activate an angiogenic program in endothelial cells by a horizontal transfer of mRNA. Blood 110: 2440-2448, 2007.

11. Mause SF and Weber C: Microparticles: Protagonists of a novel communication network for intercellular information exchange. Circ Res 107: 1047-1057, 2010.

12. Al-Nedawi K, Meehan B and Rak J: Microvesicles: Messengers and mediators of tumor progression. Cell Cycle 8: 2014-2018, 2009.

13. Breakefield XO, Frederickson RM and Simpson RJ: Gesicles: Microvesicle 'cookies' for transient information transfer between cells. Mol Ther 19: 1574-1576, 2011.

14. Antonyak MA, Li B, Boroughs LK, Johnson JL, Druso JE, Bryant KL, Holowka DA and Cerione RA: Cancer cell-derived microvesicles induce transformation by transferring tissue transglutaminase and fibronectin to recipient cells. Proc Natl Acad Sci USA 108: 4852-4857, 2011.

15. Tasca CI, Dal-Cim T and Cimarosti $\mathrm{H}$ : In vitro oxygen-glucose deprivation to study ischemic cell death. Methods Mol Biol 1254: 197-210, 2015.

16. Brunt KR, Hall SR, Ward CA and Melo LG: Endothelial progenitor cell and mesenchymal stem cell isolation, characterization, viral transduction. Methods Mol Med 139: 197-210, 2007.

17. Rosell A, Arai K, Lok J, He T, Guo S, Navarro M, Montaner J, Katusic ZS and Lo EH: Interleukin-lbeta augments angiogenic responses of murine endothelial progenitor cells in vitro. J Cereb Blood Flow Metab 29: 933-943, 2009.

18. Samaan S, Khella HW, Girgis A, Scorilas A, Lianidou E, Gabril M, Krylov SN, Jewett M, Bjarnason GA, El-said H and Yousef GM: miR-210 is a prognostic marker in clear cell renal cell carcinoma. J Mol Diagn 17: 136-144, 2015.

19. Livak KJ and Schmittgen TD: Analysis of relative gene expression data using real-time quantitative PCR and the 2(-Delta Delta C(T)) method. Methods 25: 402-408, 2001.

20. Yang N, Li D, Jiao P, Chen B, Yao S, Sang H, Yang M, Han J, Zhang Y and Qin S: The characteristics of endothelial progenitor cells derived from mononuclear cells of rat bone marrow in different culture conditions. Cytotechnology 63: 217-226, 2011.

21. Yao W, Firth AL, Sacks RS, Ogawa A, Aguer WR, Fedullo PF, Madani MM, Lin GY, Sakakibara N, Thistlethwaite PA, et al: Identification of putative endothelial progenitor cells (CD34+ CD133+ Flk-1+) in endarterectomized tissue of patients with chronic thromboembolic pulmonary hypertension. Am J Physiol Lung Cell Mol Physiol 296: L870-L878, 2009.

22. Morel O, Jesel L, Freyssinet JM and Toti F: Cellular mechanisms underlying the formation of circulating microparticles. Arterioscler Thromb Vasc Biol 31: 15-26, 2011

23. Bruno S, Grange C, Deregibus MC, Calogero RA, Saviozzi S, Collino F, Morando L, Busca A, Falda M, Bussolati B, et al: Mesenchymal stem cell-derived microvesicles protect against acute tubular injury. J Am Soc Nephrol 20: 1053-1067, 2009.

24. Losordo DW and Dimmeler S: Therapeutic angiogenesis and vasculogenesis for ischemic disease: Part II: Cell-based therapies. Circulation 109: 2692-2697, 2004.

25. Choksy SA and Chan P: Therapeutic angiogenesis. Br J Surg 93: 261-263, 2006.

26. Müller MM and Griesmacher A: Markers of endothelial dysfunction. Clin Chem Lab Med 38: 77-85, 2000.

27. Zampetaki A, Kirton JP and Xu Q: Vascular repair by endothelial progenitor cells. Cardiovasc Res 78: 413-421, 2008.

28. Kawamoto A, Asahara T and Losordo DW: Transplantation of endothelial progenitor cells for therapeutic neovascularization. Cardiovasc Radiat Med 3: 221-225, 2002.

29. Takizawa S, Nagata E, Nakayama T, Masuda H and Asahara T: Recent progress in endothelial progenitor cell culture systems: Potential for stroke therapy. Neurol Med Chir (Tokyo) 56: 302-309, 2016

30. Kaneko Y, Tajiri N, Shinozuka K, Glover LE, Weinbren NL, Cortes L and Borlongan CV: Cell therapy for stroke: Emphasis on optimizing safety and efficacy profile of endothelial progenitor cells. Curr Pharm Des 18: 3731-3734, 2012.

31. Cantaluppi V, Gatti S, Medica D, Figliolini F, Bruno S, Deregibus MC, Sordi A, Biancone L, Tetta C and Camussi G: Microvesicles derived from endothelial progenitor cells protect the kidney from ischemia-reperfusion injury by microRNA-dependent reprogramming of resident renal cells. Kidney Int 82: 412-427, 2012. 
32. Aghajani Nargesi A, Lerman LO and Eirin A: Mesenchymal stem cell-derived extracellular vesicles for kidney repair: Current status and looming challenges. Stem Cell Res Ther 8: 273, 2017.

33. Pan Q, He C, Liu H, Liao X, Dai B, Chen Y, Yang Y, Zhao B, Bihl J and Ma X: Microvascular endothelial cells-derived microvesicles imply in ischemic stroke by modulating astrocyte and blood brain barrier function and cerebral blood flow. Mol Brain 9: 63, 2016.

34. Aliotta JM, Pereira M, Johnson KW, de Paz N, Dooner MS, Puente N, Ayala C, Brilliant K, Berz D, Lee D, et al: Microvesicle entry into marrow cells mediates tissue-specific changes in mRNA by direct delivery of mRNA and induction of transcription. Exp Hematol 38: 233-245, 2010.

35. Eulalio A, Huntzinger E and Izaurralde E: Getting to the root of miRNA-mediated gene silencing. Cell 132: 9-14, 2008.

36. Chekulaeva $M$ and Filipowicz W: Mechanisms of miRNA-mediated post-transcriptional regulation in animal cells. Curr Opin Cell Biol 21: 452-460, 2009.

37. Li Q, Yu P, Zeng Q, Luo B, Cai S, Hui K, Yu G, Zhu C, Chen X, Duan $M$ and Sun X: Neuroprotective effect of hydrogen-Rich saline in global cerebral ischemia/reperfusion rats: Up-regulated tregs and down-regulated miR-21, miR-210 and NF- $\mathrm{B}$ expression. Neurochem Res 41: 2655-2665, 2016.

38. Guo S, Bai R, Liu W, Zhao A, Zhao Z, Wang Y, Wang Y, Zhao W and Wang W: MicroRNA-210 is upregulated by hypoxia-inducible factor- $1 \alpha$ in the stromal cells of giant cell tumors of bone. Mol Med Rep 12: 6185-6192, 2015.

39. Pulkkinen K, Malm T, Turunen M, Koistinaho J and Ylä-Herttuala S: Hypoxia induces microRNA miR-210 in vitro and in vivo ephrin-A3 and neuronal pentraxin 1 are potentially regulated by miR-210. FEBS Lett 582: 2397-2401, 2008.

40. Chen Z, Hu Z, Lu Z, Cai S, Gu X, Zhuang H, Ruan Z, Xia Z, Irwin MG, Feng D and Zhang L: Differential microRNA profiling in a cellular hypoxia reoxygenation model upon posthypoxic propofol treatment reveals alterations in autophagy signaling network. Oxid Med Cell Longev 2013: 378484, 2013.
41. Chan SY and Loscalzo J: MicroRNA-210: A unique and pleiotropic hypoxamir. Cell Cycle 9: 1072-1083, 2010.

42. Liu LL, Li D, He YL, Zhou YZ, Gong SH, Wu LY, Zhao YQ, Huang X, Zhao T, Xu L, et al: miR-210 protects renal cell against hypoxia-induced apoptosis by targeting HIF-1 alpha. Mol Med 23: 258-271, 2017.

43. Cicchillitti L, Di Stefano V, Isaia E, Crimaldi L, Fasanaro P, Ambrosino V, Antonini A, Capogrossi MC, Gaetano C, Piaggio G and Martelli F: Hypoxia-inducible factor 1- $\alpha$ induces miR-210 in normoxic differentiating myoblasts. J Biol Chem 287: 44761-44771, 2012.

44. Zhang Z, Sun H, Dai H, Walsh RM, Imakura M, Schelter J, Burchard J, Dai X, Chang AN, Diaz RL, et al: MicroRNA miR-210 modulates cellular response to hypoxia through the MYC antagonist MNT. Cell Cycle 8: 2756-2768, 2009.

45. Fasanaro P, D'Alessandra Y, Di Stefano V, Melchionna R, Romani S, Pompilio G, Capogrossi MC and Martelli F: MicroRNA-210 modulates endothelial cell response to hypoxia and inhibits the receptor tyrosine kinase ligand Ephrin-A3. J Biol Chem 283: 15878-15883, 2008.

46. Chan SY, Zhang YY, Hemann C, Mahoney CE, Zweier JL and Loscalzo J: MicroRNA-210 controls mitochondrial metabolism during hypoxia by repressing the iron-sulfur cluster assembly proteins ISCU1/2. Cell Metab 10: 273-284, 2009.

47. Huang X, Ding L, Bennewith KL, Tong RT, Welford SM, Ang KK, Story M, Le QT and Giaccia AJ: Hypoxia-inducible mir-210 regulates normoxic gene expression involved in tumor initiation. Mol Cell 35: 856-867, 2009.

48. Kim HW, Haider HK, Jiang S and Ashraf M: Ischemic preconditioning augments survival of stem cells via miR-210 expression by targeting caspase- 8 -associated protein 2 . J Biol Chem 284 : 33161-33168, 2009.

This work is licensed under a Creative Commons Attribution-NonCommercial-NoDerivatives 4.0 International (CC BY-NC-ND 4.0) License. 